\title{
Network Trials
}

\section{Emiliano Romagnoli}

Dipartimento di Architettura, Università degli Studi di Ferrara

emiliano.romagnoli@unifi.it

\begin{abstract}
Una ricerca svolta all'interno del Dipartimento di Progettazione dell'Architettura dell'Università degli Studi di Firenze offre l'occasione di porre al vaglio, grazie alla pratica progettuale esercitata su un caso studio, alcuni assunti teorici sulla città maturati da una certa cultura architettonica fin dalla seconda metà del secolo scorso e forse ancora scarsamente sondati. Alla base di queste teorie la rottura dell'autonomia disciplinare nella concezione dello spazio pubblico è ormai chiara: l'architettura si apre verso contenuti provenienti da altre discipline nel tentativo di recuperare l'identità dei luoghi e quel senso di appropriazione dello spazio collettivo da parte dei cittadini. Una strada difficile quella dell'eteronomia disciplinare che appare tuttavia necessario valutare nel tentativo di risolvere le conflittualità insite nella città contemporanea afflitta da separazioni sempre più marcate. Limiti, separazioni e degrado sono fenomeni che spesso si autoalimentano e quale spazialità se non quella pubblica potrebbe farsi portatrice di quei valori e soluzioni capaci di sovvertire lo stato di cose?
\end{abstract}

\section{Parole chiave}

San Salvi, spazio pubblico, periferia, rete policentrica, spazi intermedi, spazio informale

\begin{abstract}
A research carried out within the Department of Architectural Design of the University of Florence offers the opportunity to examine, thanks to the design practice exercised on a case study, some theoretical assumptions about the city matured by a part of architectural culture since the second half of last century and perhaps still poorly explored. At the basis of these theories, the break of disciplinary autonomy in the conception of public space is clear: architecture opens up to content from other disciplines in an attempt to recover the identity of places and that sense of appropriation of collective space by citizens. The disciplinary heteronomy is a difficult road but however it seems to be necessary in the attempt to resolve the conflicts inherent in the contemporary city plagued by increasingly marked separations. Limits, separations and degradation are phenomena that are often self-feeding and which space, if not the public one, could be the bearer of those values and solutions able to subvert the state of things?
\end{abstract}

\section{Keywords}

San Salvi, public space, suburb, polycentric net, in-between spaces, informal space 
Il concetto di spazio pubblico e la sua definizione affonda le sue radici nell'idea stessa di città classicamente intesa che vede la sua ossatura portante proprio nel sistema di luoghi interni ed esterni dedicati alla collettività e che sono in relazione fra loro. L'evoluzione della città degli ultimi tempi ha tuttavia determinato una crisi delle strategie e degli strumenti messi a punto nel corso di secoli di evoluzione proprio per la definizione dello spazio pubblico, una crisi che la dilatazione del fenomeno urbano non fa che acuire. Lo sviluppo di interi brani di città carenti o in alcuni casi privi di una reale spazialità pubblica ha prima determinato e poi esasperato la separazione fra centro e periferia e questa non è altro che una delle tante dicotomie che oggi affliggono i tessuti delle città. È nel tentativo di un superamento di queste dicotomie che già dal secolo scorso si ricercano e sperimentano soluzioni capaci di risarcire le fratture prodotte da uno sviluppo distratto, ridonare dignità ai luoghi e maggiore qualità alla vita degli abitanti; il lavoro di Aldo van Eyck, a partire dagli anni '50, appartiene a questo ordine di esperienze.

A. van Eyck, in riferimento alle teorie di Kevin Lynch sulla rete policentrica (Polycentric Net) arriva a definire la città come "open-ended pattern", ovvero una città con un disegno dotato di un proprio carattere, ma non finito, sul quale si può e soprattutto si deve continuare a ragionare attraverso il progetto.
Ancora oggi quello dell'open-ended pattern appare un concetto particolarmente ricco di significato perché pone l'attenzione sul fatto che occorre tornare a pensare la città perché questa è in continua crescita e mutazione. L'idea di città che emerge con la polycentric net rivela uno spostamento di attenzione dal consueto centro che coincide con uno spazio pubblico monumentale ad un'idea di molteplicità di centri che s'identificano con degli spazi informali, capaci di creare una particolare concentrazione d'intensità all'interno di un luogo. Occorre sottolineare che il pensiero sulla città di A. Van Eyck accoglie i principi moderni, tuttavia ha come punto di partenza l'isolato considerato come organismo unitario ed un'idea di spazio pubblico inteso come spazio informale, che diventa continuo attraverso il progetto degli spazi "in-between". A cavallo fra la dimensione pubblica e privata dell'abitare gli spazi in-between non solo garantiscono continuità alla rete policentrica ma gli consentono anche di assumere una precisa connotazione formale.

Ma forse non basta perché nella ricerca della fondamentale continuità della rete A. van Eyck finisce inevitabilmente per coinvolgere anche lo spazio strada richiamando in questo caso le teorie dei coniugi Alison e Peter Smithson. Come noto, negli stessi anni dell'architetto olandese, anche gli Smithson lavorano al tema dello spazio pubblico nel- 
tato di una propria identità. E' altrettanto noto come le teorie esposte dagli Smithson al CIAM 9 ad Aix-en-Provence nel 1953, contribuirono a determinare quella "prima incrinatura nella solidità teoretica del movimento moderno" (Smithson e Smithson, 1971, p. 8) confluendo successivamente nell'azione del Team $X^{1}$ fino a raggiungere una portata di scala internazionale. Appare chiaro quindi come a partire dal secondo dopoguerra all'interno del dibattito architettonico, il concetto di spazio pubblico inizia a spingersi oltre il limite stabilito dalle regole della città classica fino ad interagire con la dimensione privata, mettendo luce su una dimensione nuova intermedia tra la dimensione pubblica e quella privata avvalendosi anche di una nuova concezione dello spazio strada.

Questo scritto, a partire da un'esperienza di ricerca condotta all'interno dell'Università degli studi di Firenze e a partire da un'analisi delle teorie sullo spazio pubblico di A. Van Eyck e degli Smithsons, esplora la possibilità di intervenire sui tessuti della città contemporanea ripensando proprio la definizione dello spazio pubblico in un contesto di rigenerazione urbana di un'area altamente specialistica posta
La ricerca dal titolo "Cenesi, trasformazione e rigenerazione dei grandi complessi urbani radi strutturati con particolare riferimento agli impianti a padiglioni"2 propone l'area dell'ex struttura manicomiale di San Salvi a Firenze come caso studio dove provare ad applicare una serie di strategie volte alla riqualificazione del complesso, oggi solo parzialmente occupato dagli uffici dell'Azienda Sanitaria di Firenze. L'occasione è apparsa utile per una riflessione più ampia sulla città ed in particolare proprio sul ruolo dello spazio pubblico, quello informale, di prossimità, in grado di rivitalizzare aree "immobilizzate" da decenni.

L'area di San Salvi è un complesso di padiglioni e spazi aperti progettato dall'architetto $G$. Roster per la cura mentale nella seconda metà del IXXº secolo, oggi sede della Azienda Sanitaria Firenze. Al tempo, immerso nella campagna agricola a sud-est della città, la struttura dedicata alla cura mentale era concepita a tutti gli effetti come una cittadella dai confini e margini ben definiti da mura e porte di accesso; nel corso del XX sec. la città, in piena fase di espansione, gli è cresciuta intorno, fino ad inglobarla completamente (fig. 1). La legge che in Italia ha sancito la fine degli ospedali psichiatrici è del 1978 e da allora il complesso è stato oggetto di varie trasformazioni e destinazioni funzionali, tutte comunque nell'ambito della sanità pubblica. II ribaltamento funzionale, da struttura interclusa a struttura rivolta al pubblico non ha sortito particolari effetti a livello spaziale-volumetrico; la forte impronta dettata da impianto urbano introverso, la presenza di margini e confini, forse anche l'azione esercitata da una 'memoria collettiva' inizialmente non particolarmente predisposta verso le strutture costrittive, hanno fatto sì che il complesso appaia ancora oggi come un'isola urbana. Non esiste neanche una viabilità di confine che possa in qualche misura mediare il rapporto fra il complesso e il suo immediato intorno. Sul margine nord dell'area una serie di edifici scolastici di stampo tardo razionalista voltano le 

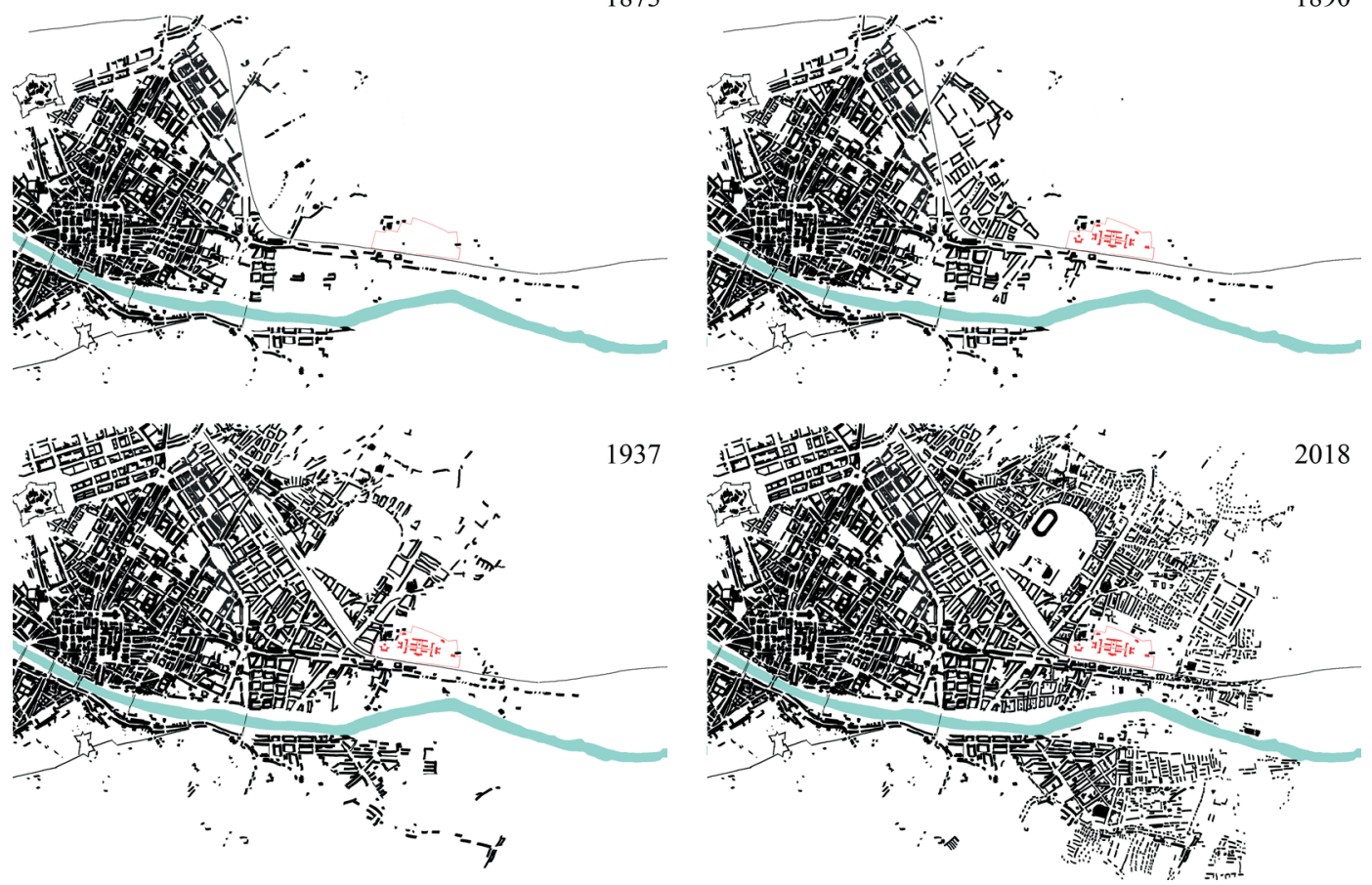

Fig. 1 - Schemi di crescita della città di Firenze nell'area sud est (disegno: Emiliano Romagnoli 2018).

spalle alla struttura sanitaria determinando di fatto una profonda frattura, a sud l'importante tracciato ferroviario che lega la città di Firenze a Roma taglia ogni relazione. L'asse est-ovest presenta margini più labili. Ad ovest, benché permanga il vecchio muro di confine a fare da barriera, è collocato il principale accesso all'area; in questa zona la trasformazione di alcune strutture sanitarie in funzioni pubbliche ha determinato una maggiore integrazione col tessuto urbano limitrofo. Ad est invece un parco pubblico dai confini sfocati lambisce il complesso sanitario. Anche su questo lato permangono muri e recinzioni ma un certo degrado coinvolge tanto gli spazi esterni quanto i padiglioni più prossimi al confine. Come indicato, il complesso venne concepito sul finire del XIX secolo in un'area allora esterna all'abitato, secondo i concetti sanitari del tempo legati alla cura mentale. A livello compositivo si tratta di un complesso di padiglioni ancorati all'asse est-ovest, in simmetria rispetto all'asse centrale nord-sud. I padiglioni hanno volumetrie semplici, rettangolari, spesso composti secondo regole aggregative altrettanto elementari a formare blocchi a 'C' o 'L'. Gli assi che strutturano la composizione sono avvalorati da un interessante sistema di percorsi e collegamenti coperti al piano terra. Percorsi ed edifici individuano delle corti circoscritte che all'occorrenza potevano essere messe in collegamento aprendo un sistema di varchi. Questo sistema di corti, poste all'intersezione dei due assi, costituisce anche il fulcro di tutta la composizione: un luogo verso cui tutte le masse sembrano tendere. Non a caso anche i blocchi a 'C' o ad 'L' rivolgono sempre la loro parte nobile verso lo spazio centrale. È così che il complesso di San Salvi dal punto di vista urbano continua ancora oggi a guardare al suo interno. I limiti del complesso, i suoi margini, appaiono oggi anche i margini della città di Firenze, quasi dei 'retri', degli spazi di risulta 
te a quella del piano terra, gli spazi esterni benché formalmente chiaramente identificati in corti e semi-corti presentano un sistema di collegamenti che possono variarne la percezione. Questi elementi rivelano un'attenta cura nel definire tutti quegli spazi di confine fra l'interno e l'esterno dei fabbricati. Forse è anche l'ingerenza nella fase di progetto di un'altra disciplina, quella della psichiatria, a contribuire a generare questo grado di complessità, sta di fatto che la continua ibridazione fra interno e esterno genera un sistema articolato di spazi a più livelli difficilmente rintracciabile in strutture analoghe. La città cresciuta intorno al complesso sanitario di san Salvi è la città del secondo dopoguerra sviluppatasi sulla base di indici e standard urbanistici, priva di memoria e priva di una reale riflessione sullo spazio collettivo. In questo tipo di città le piazze non sono altro che crocevia di traffico, le strade non sono altro che collegamenti carrabili fra due punti. E' così che, se pur posti a contatto, i due brandelli di città, appaiono oggi indifferenti l'uno all'altro. Inoltre, è forse proprio questa incomunicabilità fra le parti la principale causa della persistenza di quei margini sopra citati che evidentemente non sono solo muri, limiti fisici, ma in qualche misura anche limiti della cultura dello spazio urbano. Il progetto di riqualificazione per San Salvi parte te per dar forma ad un nuovo spazio pubblico di reIazione. Non volumi costruiti, ma solo spazi aperti, spazi informali capaci per quanto possibile di fare trama, al fine di stabilire un nuovo sistema di relazioni capace di restituire ricchezza e complessità a questo brano di città.

Una serie di fattori quali la mancanza nel quartiere di uno spazio significativo di carattere pubblico, la forte connotazione radiocentrica del complesso di San Salvi, la presenza di profondi e marcati limiti, l'insistenza sul bene di un vincolo storico artistico, la volontà della proprietà di mantenere le funzioni sanitarie all'interno della viabilità anulare, sono stati tradotti nel progetto in un sistema di luoghi e percorsi pubblici e semipubblici.

L'anello viario previsto dall'arch. Roster intorno al nucleo di San Salvi diviene un percorso multifunzionale e alla sede carrabile è associato un percorso dolce, per pedoni e biciclette. Nuovi varchi e accessi vengono aperti nelle murature di confine al fine di garantire una maggiore permeabilità dell'area seguendo tracciati storici caduti in disuso. Nella fascia compresa fra l'anello carrabile e i limiti del complesso sanitario sono organizzati una serie di spazi dedicati allo sport, all'educazione, spazi per eventi, spazi che nella loro organizzazione, a partire dall'impianto radiocentrico, ricercano allineamenti e corrispondenze con il tessuto della città circostante. In sostanza il progetto cerca di ribaltare la situazione attuale e trasformare ciò che oggi divide ed esclude in un qualcosa che unisce e include e lo fa cercando di bilanciare la forte impostazione introversa del complesso dando più peso al suo perimetro attraverso la continuità del sistema di spazi esterni (figg. 2, 3). Lo spazio pubblico, convenzionalmente pensato al 'centro', si sposta sul bordo dell'area alla ricerca di un diretto contatto con la città per ristabilire quella connessione visiva fra privato e pubblico così utile allo sviluppo di quel senso di appropriazione e identificazione di una comunità con il proprio territorio (figg.4, 5). Al centro gli edifici recuperati e destinati a funzioni sanitarie, scolastiche, uni- 


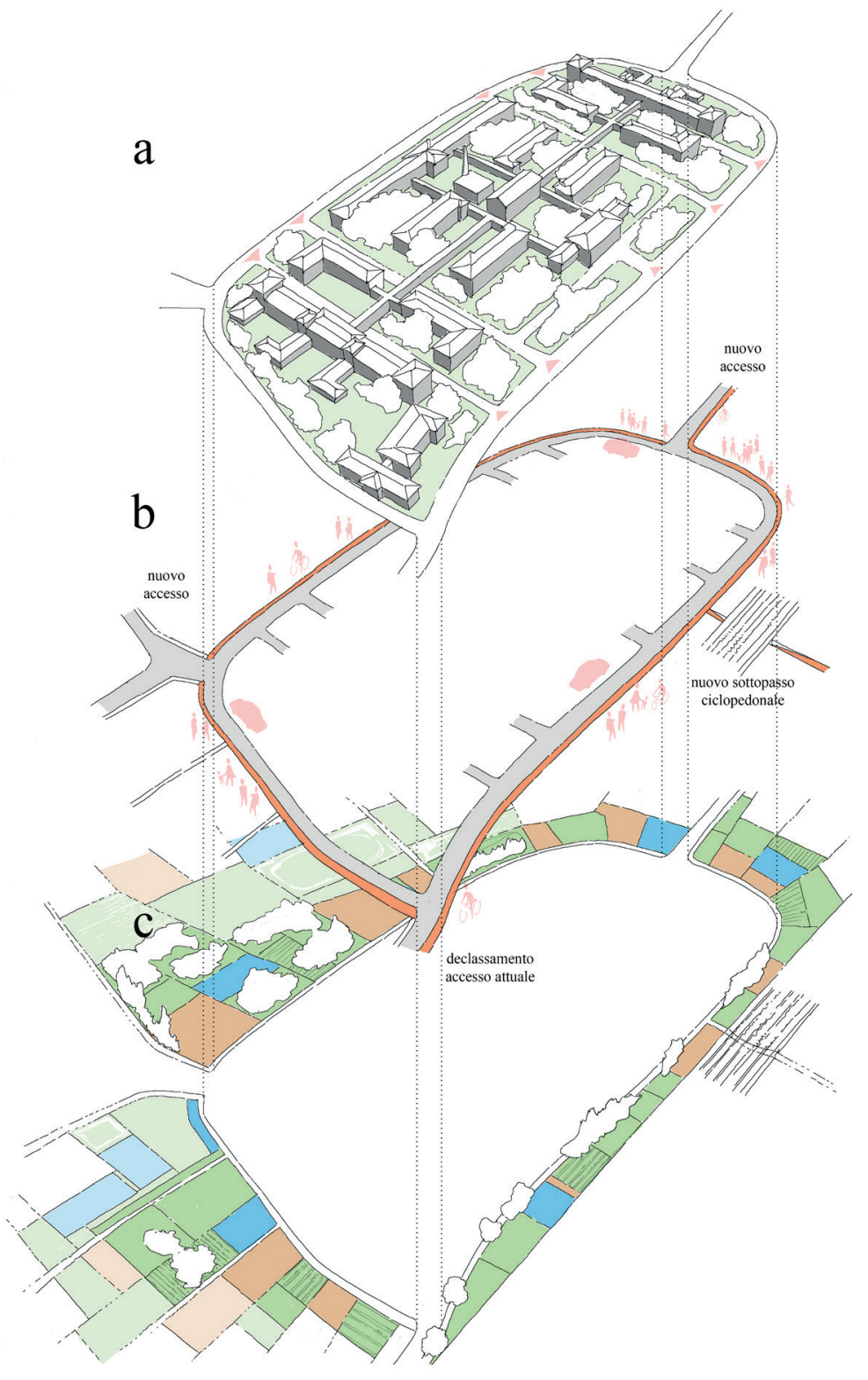

Fig. $\mathbf{2}$ - Concept di progetto,

a. Restauro della forma architettonica originale, b. Percorso anulare di collegamento, c. Anello aree di relazione (disegno: Emiliano Romagnoli 2018). 


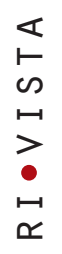

(AT) in

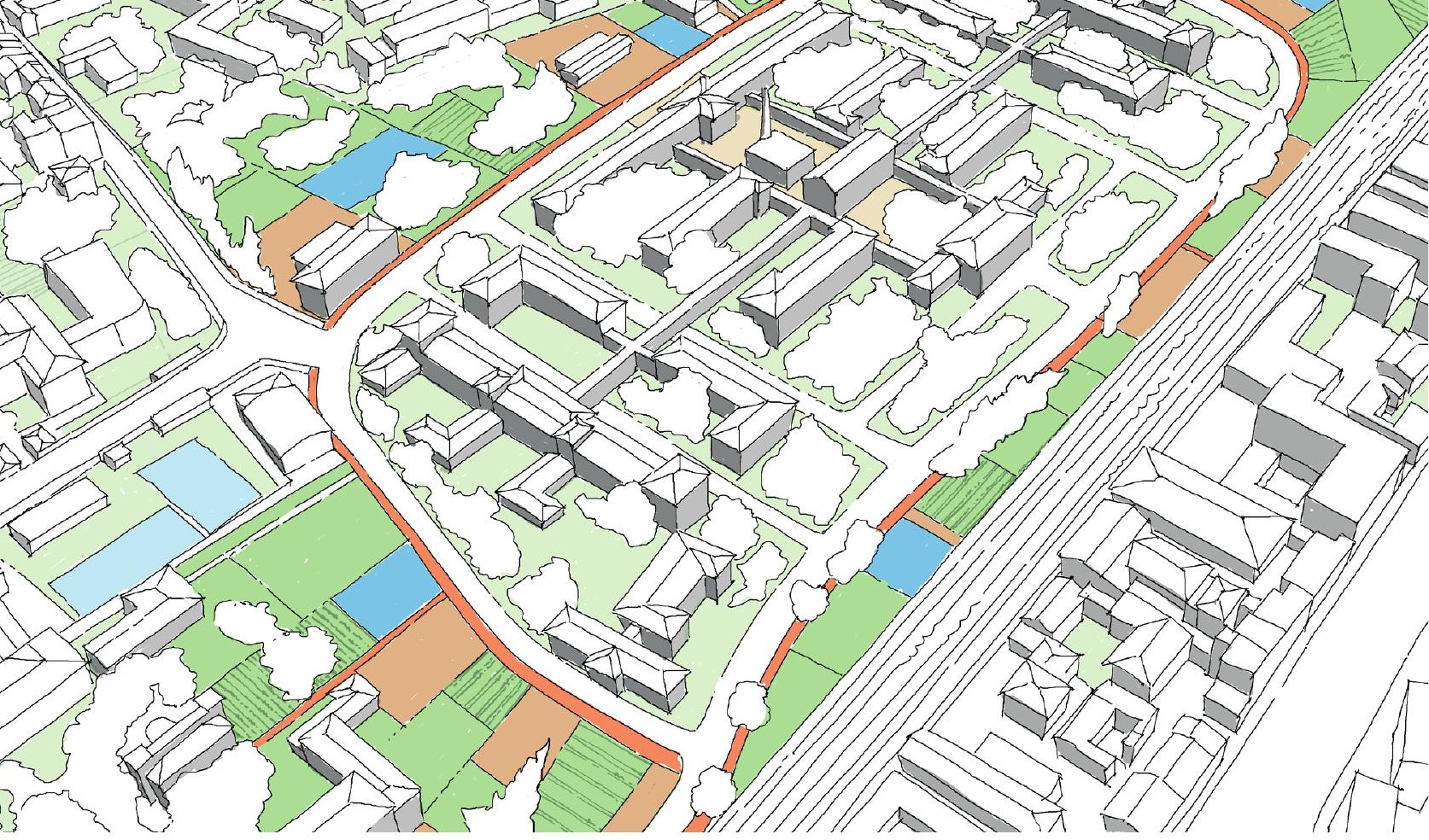

Fig. 3 - Vista aerea di progetto

(disegno: Emiliano Romagnoli 2018). 


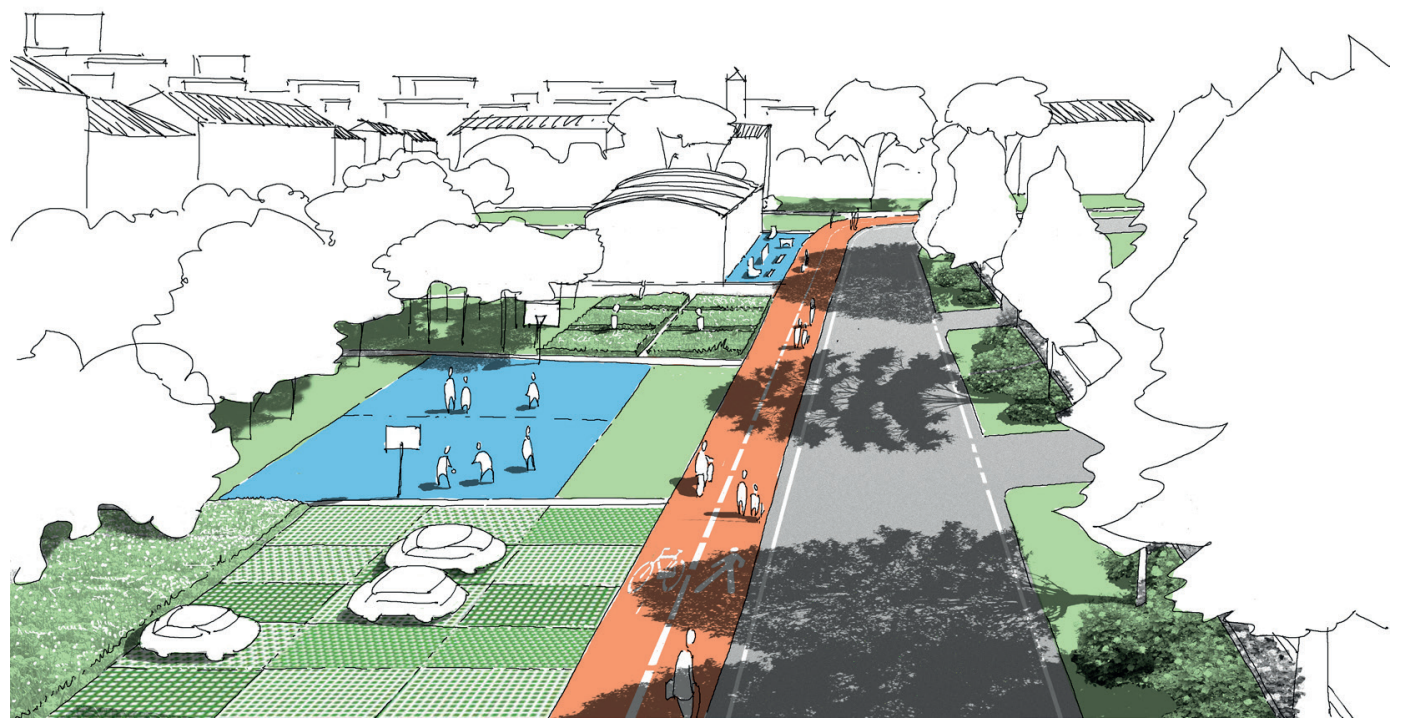

Fig. 4 - Vista aerea di progetto della porzione ovest del complesso (disegno: Emiliano Romagnoli 2018).

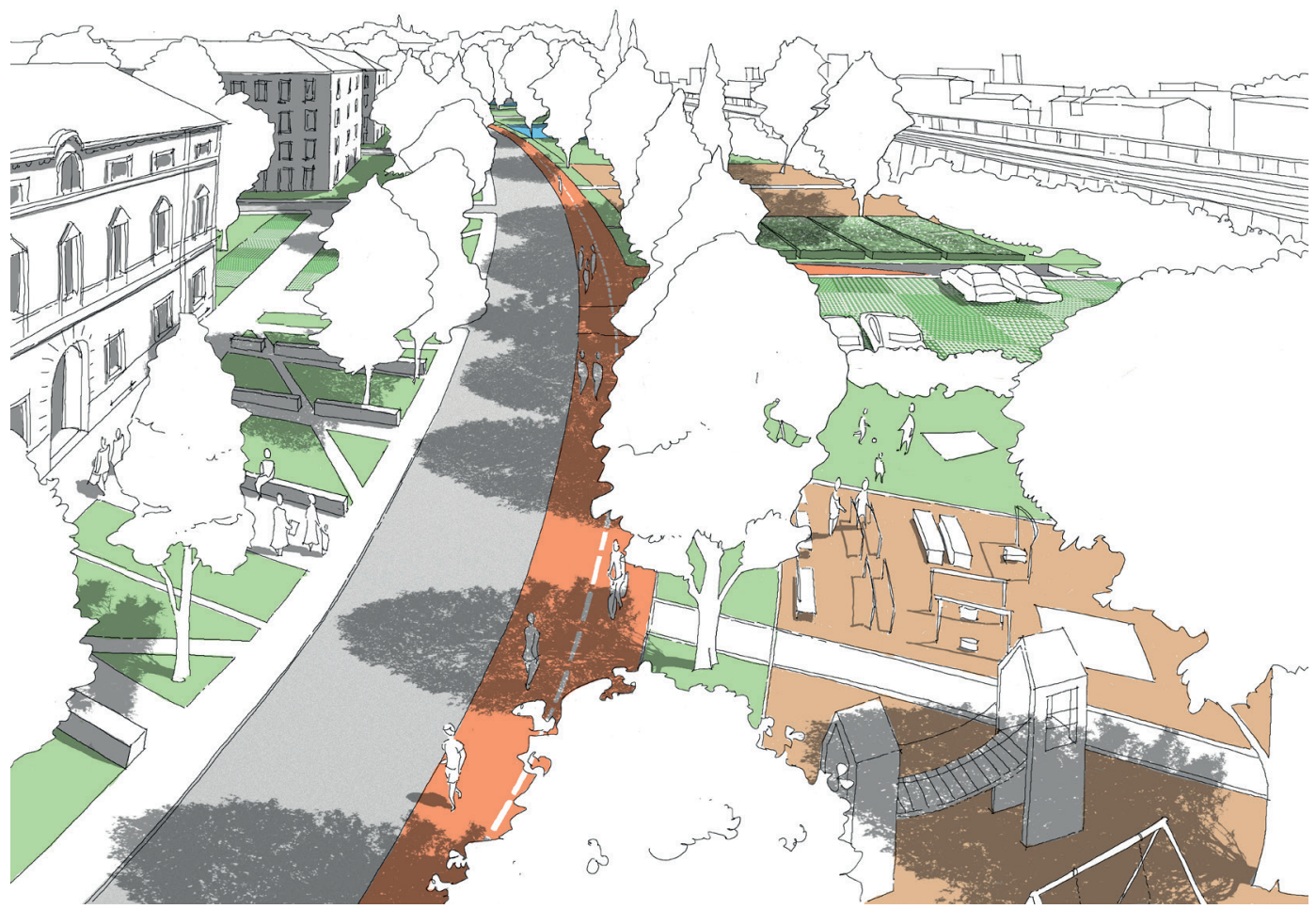

Fig. 5 - Vista aerea di progetto della porzione sud del complesso (disegno: Emiliano Romagnoli 2018). 
versitarie e residenziali continuano a guardare verso il sistema di corti interne tradotte nel progetto in spazi semipubblici a diretto contatto con l'anello esterno. L'unico percorso esistente di collegamento dell'anello con la viabilità pubblica, viene declassato poichè di sezione troppo contenuta e periferica e destinato ad ospitare il mercato rionale. Al suo posto vengono aperti due nuovi collegamenti carrabili a nord e ad est del complesso. II primo riabilita un vecchio accesso murato, il secondo sfrutta un vecchio asse agricolo che ha, per secoli, servito l'area prima del cambio di destinazione. I percorsi ciclopedonali previsti dal piano urbanistico della città vengono prolungati all'interno del complesso e raccordati attraverso il percorso anulare. Gli spazi esterni e le attività previste nella fascia compresa fra il percorso anulare e il confine dell'ex ospedale psichiatrico sono di diverso tipo:

1. Aree per la sosta carrabile e ciclabile. Le aree fra loro sono sempre in stretto contatto in maniera da agevolare il cambio di sistemi di mobilità.

2. Aree dedicate ad attività sportive come campi per il basket, il calcetto, piste per skateboard e spazi attrezzati per la ginnastica dolce rivolta alla terza età.

3. Aree dedicate ad attività educative e meditative.

4. Aree ludiche attrezzate con giochi sia per bambini che per persone adulte.

5. Aree pavimentate multifunzionali diffuse per tutto il perimetro del percorso anulare, dotate di allacci a sottoservizi in maniera da renderle utilizzabili anche a venditori di strada.

6. Aree verdi. Tutto il verde monumentale viene mantenuto e potenziato con nuove piantumazioni autoctone.

La localizzazione delle diverse aree è stata pensata seguendo alcuni criteri di prossimità fra i quali lo scambio culturale e intergenerazionale. Non esi- vità piuttosto che ad un'altra quanto piccoli gruppi di aree con specifiche vocazioni disseminate lungo tutto il percorso anulare in maniera da incentivare il movimento e lo spostamento delle persone e dunque l'incontro (fig. 6). Dietro quest'idea di sistema di spazi informali c'è, ancora una volta, il pensiero $A$. van Eyck e le diverse sperimentazioni di questa teoria svolte in anni recenti per mano di Liane Lefaivre e Henk Döll ("Ground up city")3.

Nel progetto per San Salvi c'è anche un altro tema che era già centrale nel pensiero di Aldo van Eyck ed è la questione dell'identità. I luoghi, secondo A. Van Eyck, assumono una specifica identità attraverso la complessità di caratteristiche e di valori che acquisiscono in relazione al contesto all'interno del disegno generale cui appartengono, e quindi all'interno della città. A. van Eyck ritiene che ogni luogo possiede un'immagine di sé immediatamente riconoscibile e concepisce la relazione come un luogo in-between, un luogo dove molte cose s'incontrano. Per A. van Eyck l'in-between è una sequenza di luoghi dalla scala della casa a quella della città, dal centro alla periferia, un articolato sistema di luoghi, ciascuno dotato di una propria identità. In questo senso l'articolazione dello spazio sulla soglia diviene l'aspetto di maggiore rilievo, poiché in relazione a come lo spazio viene articolato si conferisce una certa plasticità ai luoghi che è determinante ai fini della sua percezione.

Alla luce di questa ricerca teorica, occupare le corti interne del complesso di San Salvi con una spazialità pubblica sarebbe stato contraddittorio perchè ancora una volta lo spazio pubblico sarebbe coinciso con un centro dalla forma e dimensioni stabilite e soprattutto perché la spazialità pubblica non avrebbe ritrovato un diretto e necessario contatto con la città. Non solo, occupare le corti interne avrebbe anche significato la parziale perdita di quel principio di autonomia culturale e funzionale che definisce anche uno dei tratti identitari del Complesso di San Salvi come di molti altri complessi radi a padiglione. 

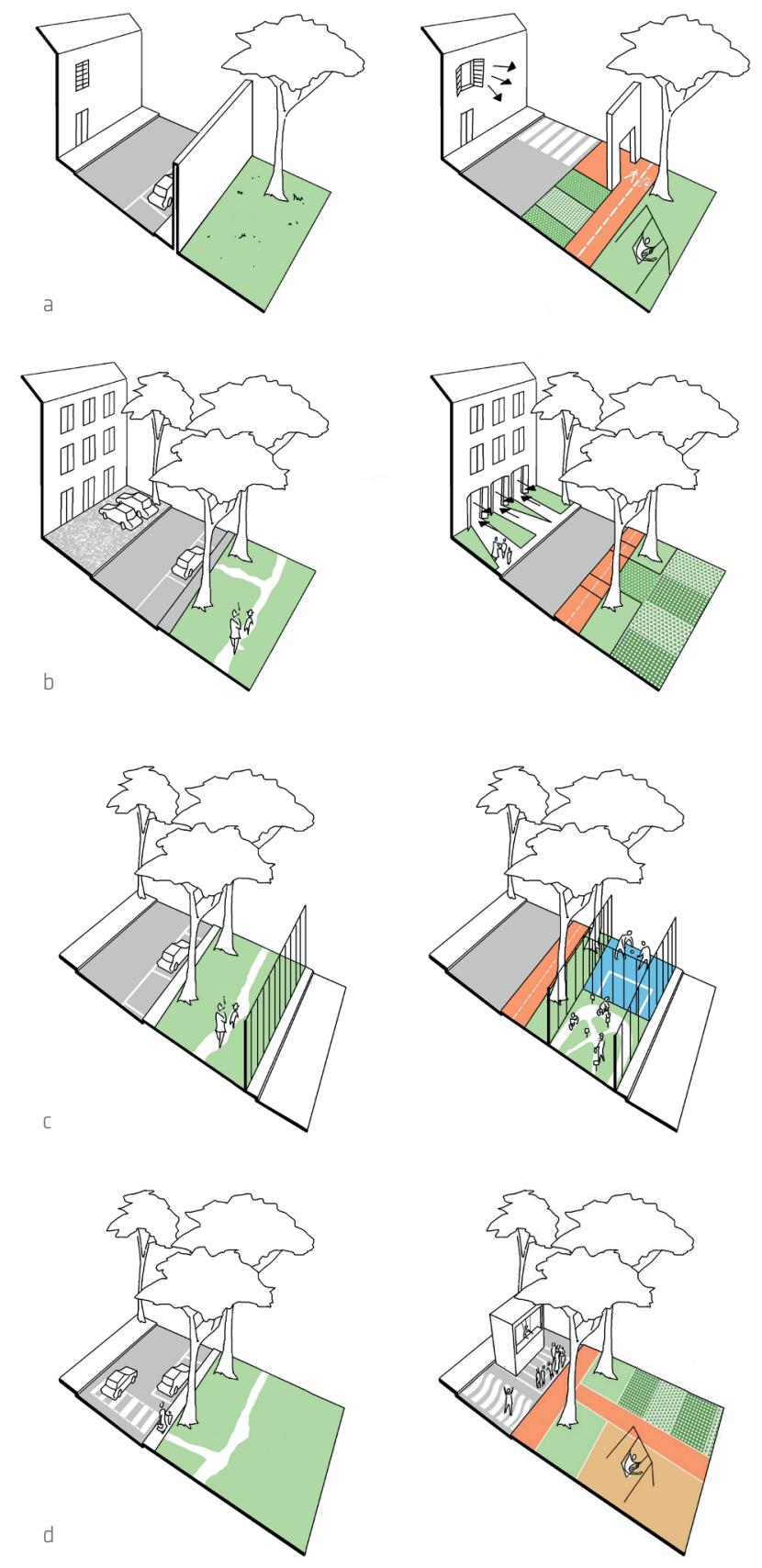

Fig. 6 - Strategie per la riqualificazione delle aree utili alla definizione della nuova spazialità pubblica. Sulla sinistra è rappresentata la condizione attuale e sulla destra le relative strategie di progetto per: a. riqualificazione di margini in presenza di muri di confine, b. riqualificazione degli accessi agli edifici del nucleo storico del complesso, c. riqualificazione di aree con limiti ineludibili per questioni normative e di sicurezza, d. riqualificazione dello spazio strada nell'anello interno (disegno: Emiliano Romagnoli 2018). 
Anche il percorso anulare di San Salvi è pensato come spazio flessibile capace di adeguarsi alle diverse esigenze e chiudibile al traffico veicolare in giornate compatibili con l'attività degli uffici al fine di favorire un'appropriazione dello spazio da parte della collettività; l'educazione alla viabilità, i mercati settimanali, eventi e fiere, sono solo alcuni degli usi che potrebbero essere previsti. In sostanza ciò che il progetto vuole affermare con forza è la riconquista dello spazio strada pensato non più come sede esclusiva dell'auto ma alla stessa stregua di tutte le altre aree dell'anello che caratterizza l'impianto di San Salvi, ovvero come spazio di relazione. Ed anche in questo caso il progetto riprende alcune riflessioni appartenenti alla ricerca teorica degli Smithsons esplorando la possibilità di ritrovare la multifunzionalità dello spazio strada, uno spazio che liberato dalla schiavitù dell'auto torni, come nella città antica, ad essere luogo di incontro dove poter stare invece che semplicemente passare.

Nel progetto di recupero del complesso di San Salvi affiorano in sostanza concetti propri ad alcune teorie sulla città sviluppate nei decenni passati ma forse ancora scarsamente sondati, concetti che, applicati a questo caso studio specifico hanno portato a rivalutare nei processi di rigenerazione urbana quelle aree usualmente considerate marginali, dove le diversità poste a stretto contatto confliggono, generando spesso degrado fisico e sociale.

Prende forma così, nel progetto di ricerca, l'idea di uno spazio pubblico come sorta di collante, capace di ri- vorire la nascita di nuovi sistemi di relazione: uno spazio che in definitiva torna ad unire ed includere.

In tutto questo, nell'ambito del dibattito sullo spazio pubblico, un sempre più imminente traguardo attende la collettività e cioè la sfida offerta dalla mobilità e della riconversione di tutti quegli spazi ad essa connessi; questi spazi richiedono un risarcimento in termini anche ambientali oltre che di senso (fig. 7).

A San Salvi, infatti, un ulteriore pensiero riguarda il verde la cui conservazione è questione ormai consolidata oltre ogni dubbio, ma qui, come in altri complessi simili, si carica forse di un ulteriore significato oltre quello ambientale. Come in parte indicato questo tipo di interventi riflettono l'azione di sezione e ricomposizione svolta sul corpo dell'architettura ad opera della cultura Ottocentesca e prima ancora Illuminista, dove quel principio di autonomia del tutto e delle sue singole parti (Kaufmann, 1973) è particolarmente evidente. Ecco, in quella necessaria distanza fra le parti, per oltre un secolo, ha talvolta prosperato una natura che ha assunto tratti monumentali e che, cresciuta intorno a quei volumi, si è ad essi in qualche maniera conformata fino a tradursi in una sorta di amalgama che tiene insieme e senza la quale queste strutture tornerebbero al dominio del pensiero astratto che le ha generate. $\dot{E}$ anche in questa natura che oggi risiede l'identità di questo tipo di complessi ed è questa natura che il progetto di ricerca vuole conservare. È proprio nel risarcimento in termini di qualità ambientale ed identità dello spazio pubblico la forza del progetto di ricerca elaborato, senza il quale San Salvi non 


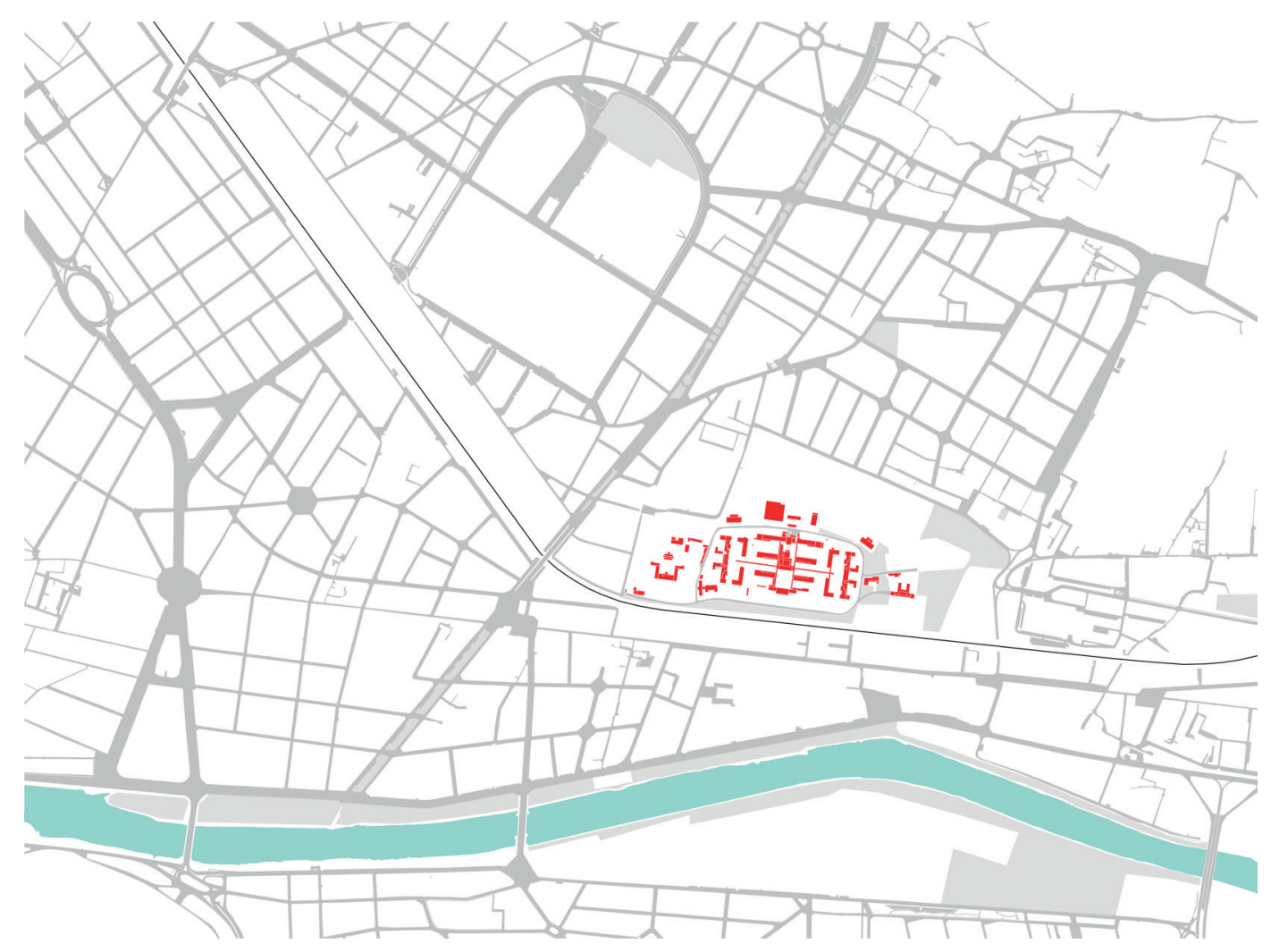

Fig. 7 - Forma dello spazio pubblico nella porzione est della città di Firenze; nel disegno sono evidenziati solo gli spazi per la mobilità carrabile in grigio e gli spazi esclusivamente pedonali in grigio chiaro (disegno: Emiliano Romagnoli 2021).

rimarrebbe altro che uno dei tanti brani isolati di cui la città contemporanea è tristemente ricca.

Si chiarisce così una visione del recupero dell'ex complesso manicomiale che in estrema analisi non pone il suo nucleo storico al centro dell'attenzione, almeno a livello teorico esso è considerato alla stessa stregua della città che gli è cresciuta intorno e quindi al pari di tutte le diversità limitrofe. II nuovo centro, se di centro si può parlare, diviene un sistema di spazi pubblici di relazione che solo nel rispetto alle preesistenze trova una conformazione anulare, spazi sorti laddove erano presenti limi$\mathrm{ti}$, separazioni, degrado e che mirano a riqualificare l'intero quartiere.

In conclusione il progetto di ricerca rivela come nel recupero dei complessi radi a padiglione non sempre elevare le spazialità intercluse fra i diversi volumi, al rango di spazi pubblici rappresenta una soluzione consona al rispetto dell'identità del luogo ed alla riqualificazione dell'immediato intorno, non sempre avvalersi di quelle spazialità concepite e definite in altri tempi rappresenta una soluzione capace di garantire quella continuità dello spazio pubblico così utile al recupero della forma della città. Sul segno tracciato da alcune esperienze dei decenni passati il progetto di ricerca, invece, ribadisce l'importanza di un ripensamento dello spazio oggi dedicato alla mobilità e si interroga su quale ruolo possano assumere tutte quelle aree considerate marginali, sospese fra la dimensione pubblica e privata dell'abitare, nei processi di riqualificazione urbana. 


\section{Note}

${ }^{1}$ II Team X è nato nell'ambito degli ultimi CIAM (Congrès Internationaux d`Architecture Moderne). In particolare il gruppo ha iniziato a concretizzarsi nel 1953 allorché alcuni giovani architetti quali i coniugi Smithson, Giancarlo De Carlo, Jaap Bakema, Aldo van Eyck, Ceorges Candilis e Shadrach Woods ricevettero il compito di organizzare il successivo congresso, il decimo CIAM di Ragusa (Dubrovnik) del 1956 (da qui il numero 10 del nome). Dopo tale congresso alcuni di questi architetti formarono il comitato per organizzare lıundicesimo CIAM ad Otterlo nel 1959, che sanci la fine del glorioso organismo.

${ }^{2}$ Ricerca condotta per il Dipartimento di Progettazione dell'Architettura, Università degli Studi di Firenze dal 01/02/2018 al 31/01/2019, Area 08, ICAR/14 Composizione architettonica e urbana.

Responsabile Scientifico Prof.ssa Francesca Privitera, Assegnista di ricerca Emiliano Romagnoli.

${ }^{3}$ Nel luglio del 2005 Liane Lefevre e Henk Döll presentano alla sede NAI a Rotterdam uno studio portato avanti del Döll - Atelier voor Bouwkunst che ha per titolo "the world is my playground". Lo studio è relativo alle sperimentazioni fatte sul tema dello spazio semi-pubblico qualificato in termini di spazi gioco nelle aree di Oude Westen e Hoogvliet a Rotterdam. A fronte degli studi fatti da Liane Lefaivre sulla figura di A. van Eyck è evidente che l'esperienza si pone in continuità proprio con i playgrounds dell'architetto olandese e ne rappresenta il rinnovato valore all'interno della città contemporanea evidenziando il ritorno al disegno del fatto urbano inteso come luogo di vita collettivo. 


\section{Bibliografia}

Airoldi C. 2013, I complessi manicomiali in Italia tra Otto e Novecento, Electa, Milano.

Banham R. 2004, Architettura della seconda età della macchina, Electa Mondadori, Milano.

Banham R. 2009, Los Angeles, L'architettura di quattro ecologie, Giulio Einaudi Editori, Torino.

Cecchini A. 2007, Al centro le periferie: Il ruolo degli spazi pubblici e dell'attivazione delle energie sociali in un'esperienza didattica per la riqualificazione urbana, Franco Angeli Edizioni.

Choay F. 1965, La città utopie e realtà, Editions du Seuil.

Corleone F. 2018, Mai più manicomi, Fondazione Michelucci Press, Fiesole.

Davis M. 2004, Città morte: storie di inferno metropolitano, Giangiacomo Feltrinelli Editore, Milano.

Foucault M. 1998, Eterotopie, Feltrinelli, Milano.

Cenise F. 2011, Strumenti Operativi per la Progettazione urbana in Olanda e in Italia (tesi di dottorato) Univesità degli Studi di Firenze, Facoltà di Architettura, non pubblicato.

Iacoboni M. 2008, I neuroni specchio: come capiamo ciò che fanno gli altri, Bollati Boringhieri Editore, Torino.

Jacobs J. 2009, Vita e morte delle grandi città. Saggio sulle metropoli americane, Giulio Einaudi Editori, Torino.

Kufmann E. 1973, Da Ledoux a Le Corbusier: Origini e sviluppo dell'architettura autonoma, Gabriele Mazzotta Editore, Milano.

Lefaivre L. 2002, Aldo van Eyck: the playgrounds and the city, NAI Publishers, Rotterdam.

Ligtelijn V., Strauven F. (a cura di) 2008, The child, the city and the artist: an essay on architecture : the in-between realm / Aldo van Eyck, SUN Editore.
Lippi D. 1996, San Salvi storia di un manicomio, Olschki, Firenze.

Lynch K. 2006, L'immagine delle città, Marsilio Editori, Venezia.

Rossi A. 1987, L'architettura della città, Marsilio Editori, Padova.

Smithson A., Smithson P. 1971, Struttura Urbana, Studidi Alison e Peter Smithson, Officine Grafiche Calderini, Bologna.

Spirito G. 2015, In-between places: forme dello spazio relazionale dagli anni sessanta a oggi, Quodlibet, Macerata.

Strauven F. 1998, Aldo van Eyck, the shape of relativity, Architettura \& Natura, Amsterdam. 